\title{
False-Positive RT-PCR Screening for SARS-CoV-2 in the Setting of Urgent Head and Neck Surgery and Otolaryngologic Emergencies During the Pandemic: Clinical Implications
}

\author{
Francisco J. Civantos MD FACS ${ }^{1}$ \\ ${ }^{1}$ University of Miami Miller School of Medicine
}

May 19, 2020

\begin{abstract}
Background: There are no reports regarding false positive reverse transcriptase polymerase chain reaction (RT-PCR) for novel coronavirus in preoperative screening.

Methods: Pre-operative patients had one or two nasopharyngeal swabs, depending on low or high risk of viral transmission. Positive tests were repeated.

Results: Forty-three of 52 patients required 2 or more pre-operative tests. Four (9.3\%) had discrepant results (positive/negative). One of these left the COVID unit against medical advice despite an orbital abscess, with unknown true disease status. The remaining 3 of $42(7.1 \%)$ had negative repeat RT-PCR. Although ultimately considered false positives, one had been sent to a COVID-unit postoperatively, and two had urgent surgery delayed. Assuming negative repeat RT-PCR, clear chest imaging, and lack of subsequent symptoms represent the "gold standard", RT-PCR specificity was 0.97.

Conclusions: If a false positive is suspected, we recommend chest computed tomography and repeat RT-PCR. Validated immunoglobulin testing may ultimately prove useful.

Andrew P. Katz MD ${ }^{1}$, Francisco J. Civantos MD FACS ${ }^{1}$, Zoukaa Sargi MD MPH ${ }^{1}$, Jason M. Leibowitz MD FACS $^{1}$, Elizabeth Nicolli MD FACS ${ }^{1}$, Donald Weed MD FACS ${ }^{1}$, Alexander E. Moskovitz BA ${ }^{1}$, Alyssa M. Civantos $\mathrm{BA}^{2}$, David M. Andrews $\mathrm{MD}^{1}$, Octavio Martinez MD ${ }^{1}$, Giovana R. Thomas MD FACS ${ }^{1}$

${ }^{1}$ Department of Otolaryngology, University of Miami Miller School of Medicine, Miami, FL

${ }^{2}$ Perelman School of Medicine at the University of Pennsylvania, Philadelphia, PA
\end{abstract}

\section{Address correspondence and reprint request to:}

Francisco J. Civantos MD FACS

Sylvester Medical Office Building

1121 NW 14 St Rm 320

Miami, FL 33136

Phone: (305) 243-5276, E-mail: fcivanto@med.miami.edu

Brief Running Title: False positive preoperative COVID-19 tests 
Keywords: COVID-19, pandemic, head and neck surgery, RT-PCR, preoperative testing

Funding and conflicts of interests: None

Acknowledgements: The authors would like to acknowledge Alejandro Mantero PhD for assistance with statistical review.

\section{ABSTRACT}

Background: There are no reports regarding false positive reverse transcriptase polymerase chain reaction (RT-PCR) for novel coronavirus in preoperative screening.

Methods: Pre-operative patients had one or two nasopharyngeal swabs, depending on low or high risk of viral transmission. Positive tests were repeated.

Results: Forty-three of 52 patients required 2 or more pre-operative tests. Four (9.3\%) had discrepant results (positive/negative). One of these left the COVID unit against medical advice despite an orbital abscess, with unknown true disease status. The remaining 3 of $42(7.1 \%)$ had negative repeat RT-PCR. Although ultimately considered false positives, one had been sent to a COVID-unit postoperatively, and two had urgent surgery delayed. Assuming negative repeat RT-PCR, clear chest imaging, and lack of subsequent symptoms represent the "gold standard", RT-PCR specificity was 0.97.

Conclusions: If a false positive is suspected, we recommend chest computed tomography and repeat RTPCR. Validated immunoglobulin testing may ultimately prove useful.

\section{INTRODUCTION}

The World Health Organization designated the COVID-19 Coronavirus Disease outbreak as a global pandemic on March 11, 2020. ${ }^{1}$ Three days later the Surgeon General recommended that all elective surgeries be cancelled, and on March 20, 2020, elective surgeries were banned in the state of Florida. ${ }^{2,3}$ The rapid and deadly spread of this pandemic has led to a reconsideration of traditional treatment paradigms in head and neck surgery, with an emphasis on protecting both the patient as well as the treatment team. ${ }^{4,5,6}$ The virus responsible for COVID-19, SARS-CoV-2, poses a particular risk to providers involved in the care of otolaryngology patients due to examinations and surgeries involving the nasopharynx, oropharynx, and upper aerodigestive tract, which harbor high concentrations of viral particles. Instrumentation of these areas may aerosolize viral particles, further increasing the risk of infection for any member of the surgical team..$^{7,8}$

In an effort to ensure the safety of both patients and providers requiring urgent otolaryngology surgeries, the University of Miami has adopted protocols in order to triage patients prior to head and neck surgeries. ${ }^{6}$ In line with other institutions across the globe, these protocols call for preoperative testing of asymptomatic patients using reverse transcriptase polymerase chain reaction (RT-PCR) given reports of asymptomatic carriers of SARS-CoV-2 capable of transmission. ${ }^{9-16}$ Reports of the sensitivity of the RT-PCR are limited, and the studies have relatively small sample sizes; however, sensitivity is reported between $63 \%$ and $78 \% .{ }^{17-19}$ Few papers report the specificity, but specificity has been cited as high as $98.8 \% .{ }^{17}$ Sensitivity changes depending on the site of collection, with oropharyngeal swabs lower than nasopharyngeal swabs, but sputum and bronchoalveolar lavage with sensitivity reported as high as $72 \%$ and $93 \%$, respectively. ${ }^{19}$ Importantly, these numbers may differ between and even within institutions due to the wide variety of testing platforms that have different molecular targets for the virus particle. It remains unclear how sensitive and specific RT-PCR tests are for detecting SARS-CoV-2 in asymptomatic patients in the preoperative setting. 
In this article, we discuss the importance of considering the potential for false positive results when testing these patients. False positive tests carry serious implications for preoperative patients and providers and can lead to changes in patient care that would not otherwise have occurred. Our objective is to share our experiences with false positive test results during preoperative screening, discuss the implications for our patients, and offer our recommendations regarding these circumstances.

\section{METHODS}

All surgical cases scheduled at our medical campus between March 30, 2020 and April 24, 2020 were reviewed. Before surgery, these cases had been presented to and approved by a Surgical Review Committee and were deemed either emergent or urgent. Departmental policy regarding preoperative screening evolved over this time period but solidified by the second week of this study (for detailed explanation of policies, refer to Civantos et $a{ }^{6}{ }^{6}$ ). For procedures involving mucosa of the upper respiratory tract, which pose high-risk for aerosolization of virus if present, two negative tests were required preoperatively with the second test recommended within 24 hours of surgery. For surgeries not involving mucosa (i.e., low risk), only one negative preoperative test was required.

Patients were tested preoperatively for SARS-CoV-2 infection on nasopharyngeal swabs using RT-PCR with at least one RNA primer approved by the Food and Drug Administration for testing. At the beginning of the study period, tests were outsourced to LabCorp (Burlington, NC) and Quest Diagnostics (Secaucus, NJ) laboratories, with turnaround time of five to seven days. As time progressed, the process was effectively streamlined by hospital administration, laboratory administration and personnel, and the preoperative department. RT-PCR was transitioned to testing in our own hospitals, with turnaround time of four hours or less. The in-house test systems and molecular targets can be found in Table 1. Multiple platforms were kept available due to supply chain shortages with all systems during the pandemic. Obtaining testing cartridges, kits, and reagents were a challenge in South Florida from mid-March until early May. Thus, in order to ensure that our ability to run RT-PCR for SARS-CoV-2 was not interrupted, several platforms were kept available at all times.

Patients with discrepant results were those who had different results on the first two preoperative tests (i.e., one positive and one negative). All patients were closely followed perioperatively by their surgeons and members of the committee to ensure the safety of the patient and all healthcare personnel involved.

Data was analyzed using univariate analyses: percentages were used to summarize categorical outcomes and sensitivity was calculated to determine test performance. The study was granted exemption by the University of Miami Institutional Review Board.

\section{RESULTS}

In the study period, 52 patients were scheduled for surgery, of which $45(86.5 \%)$ were "high risk" procedures for SARS-CoV-2 transmission. Forty patients (76.9\%) had mucosal pathology (e.g., malignant neoplasm, infection, inflammation); 37 patients (71.1\%) carried current diagnoses of head and neck cancer, including mucosal and cutaneous malignancies. Ten patients (19.2\%) had a prior history of radiation to the head and neck. There was no significant difference in the makeup of the groups with and without discrepant results with regard to mucosal pathology, current cancer diagnoses, or history of radiation (Table 2).

Forty-three $(82.7 \%)$ patients had at least two preoperative tests performed. A total of 102 tests were performed: $52(51.0 \%)$ were for the first preoperative test, and $43(42.2 \%)$ were the second preoperative test, when applicable. An additional seven tests were performed beyond the two initial tests: 2 were for patients with 2 previously negative tests, due to unrelated surgical delays; the remaining 5 were for patients with conflicting results on the first two tests.

Of the 43 patients with more than one preoperative test, four patients $(9.3 \%)$ had discrepant results on the first two tests, with the clinical implications for each discussed below (Table 3). One of these patients left against medical advice and thus his true disease status is unknown. For the remaining three out of fortytwo patients (7.1\%), repeat RT-PCR testing was negative. They remained asymptomatic from COVID-19 
official symptomatology, ${ }^{20}$ and chest radiographs were clear without signs of interstitial pneumonia. For two of these patients, positive tests were re-reviewed by clinical pathologists, and tests were found to have achieved positivity with titers that were borderline, just over the cutoff values. All three of these patients were discussed by multi-disciplinary teams including infectious disease specialists, otolaryngologists, intensivists, pulmonologists, and pathologists, which ultimately concluded that the positive test were false positives. Assuming this determination is correct, and using prior and subsequent negative RT-PCR, chest imaging and close follow up of clinical course as the "gold standard" in the absence of a true gold standard test, the calculated specificity of this test in asymptomatic patients in the preoperative setting is 0.97 .

Patient \#1-Late-March : A 60-year-old male with buccal squamous cell carcinoma required composite oral cavity resection, neck dissection, and free flap reconstruction. At the time of this patient's surgery, testing turnaround was at least 3 days. Four days prior to scheduled surgery, the patient had a negative RT-PCR drawn as an outpatient. On the morning of surgery, a second RT-PCR was drawn due to a policy change requiring two tests before surgery. A positive result was reported on postoperative day (POD) 3. Chest computed tomography (CT) with contrast was normal. He was moved to a designated COVID-19 ward. A repeat RT-PCR was performed on POD 4 with a negative result reported on POD 5, and the patient was transferred out the designated ward. During that time, he remained asymptomatic with 2 normal chest radiographs. ${ }^{6}$

Over the next month he developed no symptoms to suggest COVID-19 and repeat a CT chest was negative.

Patient \#2 - Mid-April: An 81-year-old female was transferred for recurrent aspiration pneumonia. She had originally been treated for oropharyngeal squamous cell carcinoma by definitive chemoradiation, followed by salvage pharyngectomy and tongue base resection with neck dissection, and radial forearm free flap reconstruction. Her postoperative course after discharge had been complicated by a nonfunctional larynx and recurrent aspiration pneumonias, with multiple admissions to outside hospitals over several months. On her current admission to an outside facility, the decision was made for transfer to our hospital for narrow-field laryngectomy and pectoralis major flap as treatment for recurrent aspiration. Prior to transfer, she had two negative COVID-19 RT-PCR tests, and an additional negative test on the day of arrival (hospital day 1) with plans for surgery on hospital day 3. On hospital day 2, a second RT-PCR was performed per departmental protocol, and the result was positive. A third RT-PCR on the morning of hospital day 3 was negative, however surgery was cancelled due to discrepant results. Three additional tests were drawn between hospital days 3 and 7, which were all negative. Multiple chest CTs were stable or improved, although abnormal given her underlying history of recurrent aspiration pneumonia over 6 months. Discussion with our clinical pathologists revealed that the positive test was based on weakly positive titers. Given the multiple repeat negative tests, this was deemed a false positive, and surgery was performed on hospital day 7 . As a result of the positive test, surgery was delayed 4 days while the patient remained an inpatient.

Patient \#3 - Mid-to-late April: 63-year-old female with melanoma of the cheek requiring wide local excision and sentinel lymph node biopsy. Four days prior to scheduled surgery, an outpatient RT-PCR was done and resulted positive. Immediate discussion with pathology colleagues revealed a weakly positive titer, just above the level necessary to be considered positive. The positive test had been conducted at the affiliated institution, and the negative test was at her primary hospital. One option discussed was to delay surgery for three weeks anticipating possible development of COVID-19 symptoms. However, with a negative CT chest for metastatic workup in the days before surgery, the decision was to repeat two additional RT-PCR tests. These were performed the following day and were both negative. The patient was completely asymptomatic. Surgery proceeded with a three-day delay. The process was quite stressful for the patient, whose surgery was already delayed by several weeks due to the pandemic, and the reconstructive plastic surgery team had to reschedule other surgeries to accommodate the new schedule. Over the next three weeks she developed no symptoms of any type, and repeat CT chest was normal.

Patient \#4 - Early April: 20-year-old male with bilateral pansinusitis complicated by unilateral orbital abscess and progressive vision impairment with diffusely restricted extraocular movements and an intraocular pressure of $41 \mathrm{mmHg}$. He was transferred from an outside hospital without any testing or chest radiographs. 
On the day of arrival, hospital day 1, RT-PCR was performed and was negative. Intravenous antibiotics were instituted, and surgery was planned for hospital day 4 to allow for preoperative COVID-19 testing. Since sinus surgery was considered a high-risk procedure for SARS-CoV-2 transmission, a second RT-PCR was performed according to protocol and unfortunately was positive. The patient remained asymptomatic other than symptoms attributed to sinusitis and orbital abscess. Due to the positive finding, surgery was delayed for further testing, and he was transferred to a designated COVID-19 ward. Patient was uneasy about being on this ward, and subsequently left against medical advice without surgery on hospital day 5 , refusing further workup. In telemedicine follow-up three weeks later, he reported never having coronavirus-associated symptoms; however, without additional workup his true disease status is unknown. He has therefore been excluded from statistical calculations related to the test. The patient reported that his infection improved on oral antibiotics, and visual changes normalized.

\section{DISCUSSION}

Discrepancies in preoperative reverse transcriptase polymerase chain reaction (RT-PCR) screening for SARS$\mathrm{CoV}-2$ can create major concerns with regards to proceeding with otolaryngologic surgeries that are medically urgent yet risky for SARS-CoV-2 transmission. Justified fear for the safety of the patient and the healthcare providers involved in the patient's care can cause significant changes to the course of care. Lei et al. (2020) have reported that operating during the prodrome of COVID-19 can lead to life-threatening complications. False negative results are widely known to occur and are well-documented in the medical literature, however nothing has been published about false positive results. ${ }^{21}$ Therefore, when faced with patients with suspected false positive results, our department and institution as a whole were left with little guidance on how to proceed in these circumstances. It would have been dangerous to providers to simply assume the single positive result was spurious; likewise, delaying urgent surgery for three weeks for what might be a spurious positive result carried significant implications for the patient.

We turned to the expertise of several colleagues, including pathology, radiology, and infectious disease, as well as internal departmental experts who were part of the Surgical Review Committee. It seemed reasonable that if repeat testing, multiple times in some instances, was negative, we could declare patients as falsely positive and safely proceed with surgery. Chest imaging, particularly computed tomography, and subsequent clinical course also supported the determination that these were false positives. These false positives tests were seen in a clinically significant $7.1 \%$ of our patients. As part of the decision process, pathologists were able to rereview tests to report the titer levels. Rather than accepting the results reported simply as "detected" or "not detected" in the electronic medical record, we discussed suspicious cases to determine if titers were borderline positive or strongly positive. We sparked conversation with infectious disease physicians, pulmonologists, and particularly infectious disease specialists who were now specializing in the care of COVID-19 positive patients to seek expert opinions.

To our knowledge, there are no papers reporting false positive SARS-CoV-2 RT-PCR tests. Tahamtan and Ardebili (2020) discuss possible factors causing false negative results of SARS-CoV-2 RT-PCR, namely mismatches between the testing primers and viral genome or low viral loads in samples due to timing of disease or location of collection. ${ }^{22}$ False positive RT-PCR for the diagnosis of norovirus and dengue virus have been discussed, and indicate higher rates of false positives for certain viral targets. ${ }^{23-25}$ This supports repeat testing of patients using a different testing platform with a different viral target for more accurate testing when the result is questioned.

We hypothesize that mechanisms for generation of false positive results for SARS-CoV-2 may include the following:

1) pure technical artifacts where fluorescence signal is generated due to non-specific nuclease degradation of the probe probably associated with off-target probe binding. In short, a technical artifact. This is most likely associated with a weak positive signal.

2) detection of another non-SARS-CoV-2 virus/microorganism that has not yet been accounted for in the global databases used to design the primers/probes in these assays. Recall that all of these assays are new. 
3) technical cross-contamination at any point along the sample chain of testing. If a manual pipetting step is involved, it could a technologist who accidentally made an error. In some instrument configurations, it could be a sample carryover contamination event. We tend to trust robots and instruments, but they are not infallible.

Otolaryngology patients are unique in that the patient often has disease involving the mucosa in the upper aerodigestive where the SARS-CoV-2 virus resides, and from which our nasopharyngeal swabs are obtained. Changing the location in which the sample is obtained could be considered. One of our patients, who was heavily irradiated in the area swabbed, had a tracheostomy, and thus, a bronchioalveolar lavage would have been easy to obtain. However, this was not performed. There has been no previous discussion in the literature regarding whether local mucosal cancer, infection, or inflammation can affect RT-PCR testing for this disease. Individual history of radiation to the head and neck can affect saliva production which may theoretically alter the ability of nasopharyngeal swab to collect specimen. While there was no difference seen in our groups regarding mucosal pathology, cancer diagnosis, or history of radiation, our sample size is quite small and further investigation is warranted. In any case, it would seem logical that mucosal abnormalities would lead to false negatives more often than false positives due to inadequate sampling.

Chest computed tomography (CT) has never been described as a tool for screening asymptomatic preoperative patients with no history of COVID-19 exposure. It has, however, been discussed for its utility as both a sole diagnostic test and as an adjunct along with RT-PCR. Findings of COVID-19 have been reported as ground-glass opacities with segmental consolidations; these overlap with many other pulmonary disease and therefore on meta-analysis, pooled specificity and positive-predictive value is low, $37 \%$ and $1.5-30.7 \%$ respectively. ${ }^{26,27}$ Sensitivity of CT chest alone is $94-97 \%$, with a negative predictive value of 95.4 to $99.8 \%$ in reports out of China with high prevalance. ${ }^{26,28} \mathrm{Ai}$ et al. (2020) showed $75 \%$ of patients with symptoms concerning for COVID-19 and a negative RT-PCR but positive CT chest later converted to positive RT-PCR. ${ }^{28}$ Individual case reports have similarly shown the utility of chest CT as adjunct with RT-PCR to diagnose COVID-19 in patients with negative RT-PCR. ${ }^{27,29}$

There are reports of asymptomatic positive patients with classic CT chest findings for COVID-19, however these patients were known to have exposure to SARS-CoV-2. ${ }^{30,31}$ Between $70-100 \%$ of patients were found to have CT findings consistent with COVID-19, and between one-fifth and one-fourth of patients later developed symptoms of the disease. Our preoperative patients were asymptomatic with no known history of direct COVID-19 exposure; their only risk factor was living in a city during the ascending portion of the COVID-19 pandemic. Regardless, these reports provide evidence for CT chest changes in pre-symptomatic patients. It therefore seems reasonable that $\mathrm{CT}$ chest can serve as an adjunct test to help providers determine if subclinical infection could be present. This may assist with determining which of the two results is false. A negative CT chest can help reassure providers that the positive RT-PCR test is spurious and allow the surgery to proceed as scheduled.

Among our four patients with discrepant results: five additional RT-PCR tests were performed, two patients had surgery delayed 3 or 4 days each, two patients were sent to COVID-19 designated units, and one patient left against medical advice without surgery for an orbital abscess. As the weeks progressed, we became familiar with the possibility of false positive findings and had raised suspicions for when positive results might indeed reflect false positives. In the most recent patient with positive preoperative testing without symptoms (Patient \#3), pathologists recommended immediate re-testing based on the borderline titers in her test results rather than delaying surgery for weeks for a potential COVID-19 infection. We were therefore able to move swiftly and continue with the surgery, rather than delay for weeks anticipating a clinical COVID-19 infection, which a more strongly positive test might have required.

Prolonged inpatient stays pose risks for all patients at all times; however, in the midst of the pandemic there are heightened risks. Particularly for Patient \#2, who falls into a highly at-risk group due both to age and underlying pulmonary disease, the importance of a prolonged stay should not be minimized. Fortunately, we were familiar with the possibility of false positives by the time that this patient's positive test occurred, and we were able to keep her out of the COVID unit until additional testing was performed and negative 
on three further repeat tests. Placement in COVID-19 designated units, as seen with the earlier cases of Patient \#1 and \#4, creates safety concerns for both patients and providers. While COVID-specific units are appropriate and necessary for the safe treatment of COVID-positive patients, the misplacement of false positive patients in these units exposes the patients to SARS-CoV-2 at an unnecessarily high rate. Providers from the surgical team, who in ordinary circumstances would not be in these high-risk units, are also placed in an environment with increased risk of disease contraction for themselves and risk of transmission to other patients. Moreover, our young and healthy patient was so concerned about his placement in this high-risk ward that he left against medical advice despite the risk of progressive vision loss. The psychological effects of these wards on our surgical patients should not be dismissed.

At this point in time, there is no "gold standard" test to which the results of the RT-PCR can be compared, which limits the statistical validity in the reporting a specificity. We therefore favor reporting a probable false positive rate of $7.1 \%$ in lieu of specificity, until such a time that a more accurate test is available. However, if we are to use chest imaging, close clinical follow up, and expert opinion as a gold standard in the absence of a true gold standard, the specificity would be 0.97 . Classic statistical teaching is that a positive result in a highly specific test, such as in this case, should rule in the disease. However, we believe these test results must be considered as part of the broader clinical picture.

Little data exists about the use of RT-PCR tests to screen preoperative patients without symptoms of COVID-19. In the preoperative setting, the use of a screening test such as RT-PCR for SARS-CoV-2 is faulty in that screening tests have a higher accepted false positive rate, and the pre-test probability of a positive test in these patients is already low. Ideally there should be a confirmation test for positive results with higher accuracy, and perhaps, once validated, testing for immunoglobulins against SARS-CoV-2 can fill this role in the future. As the prevalence of the disease continues to increase, concern over false positives will increase as many providers become hesitant to consider a test falsely positive. Unlike false positive tests in the general population for whom this result would cause self-quarantine, unrealized false positive tests in preoperative patients can have significant clinical implications and interfere with urgent cancer surgery or other emergency surgery.

\section{CONCLUSION}

Recent experiences at our hospitals suggest that despite high specificity of RT-PCR for SARS-CoV-2, positive screening tests in the absence of other symptoms or classic findings of the disease should be rigorously verified before urgent care is delayed. It is a disservice to our patients not to remain vigilant to possible false positive tests as the resultant clinical implications can jeopardize the safety of our patients. We recommend a multidisciplinary approach for investigation, including re-evaluation of RT-PCR titers by pathologists, repeating RT-PCR on a different testing platform, and obtaining a CT of the chest, particularly for patients who do not have other chest pathology. Testing for immunoglobulins specific for SARS-CoV-2 antigens was not yet widely available at the time of these interventions, but this may prove useful as it becomes more readily available.

\section{REFERENCES}

1. Adhanom, T. WHO Director-General's Opening Remarks at the Media Briefing on COVID-10, 11 March 2020. Retrieved from https://www.who.int/dg/speeches/detail/who-director-general-sopening-remarks-at-the-media-briefing-on-covid-19-11-march-2020. Accessed May 2, 2020.

2. State of Florida, Office of the Governor, Executive Order EO 20-72. Emergency Management - Covid19 - Nonessential Elective Medical Procedures.

3. Tweet from @Surgeon General, March 14, 2020. Twitter.

4. Dong E, Du H, Gardner L. An interactive web-based dashboard to track COVID-19 in real time. Lancet Infect Dis; published online Feb 19, 2020.

5. Day AT, Sher DJ, Lee RC, et al. Head and neck oncology during the COVID-19 pandemic: Reconsidering traditional treatment paradigms in light of new surgical and other multilevel risks Oral Oncol. [Epub ahead of print, Apr 6, 2020] 
6. Civantos F., Leibowitz, JM, Arnold DJ, et al. Ethical surgical triage of head and neck cancer patients during the COVID-19 pandemic. Head Neck. [Epub ahead of print, May 1, 2020]

7. Zou L, Ruan F, Huang M, et al. SARS-CoV-2 viral load in upper respiratory specimens of infected patients. N Engl J Med. 2020;382(12):1177-1179.

8. Givi, G et al. Safety Recommendations for Evaluation and Surgery of the Head and Neck During the COVID-19 Pandemic. JAMA Otolaryngol Head Neck Surg [Epub ahead of print, Mar 21, 2020]

9. Couloigner V, Schmerber S, Nicollas R, et al. COVID-19 and ENT Surgery. European Annals of Otorhinolaryngology, Head Neck [Epub ahead of print, April 23, 2020]

10. Fakhry N, Schultz P, Morniere S, et al. French consensus on management of head and neck cancer surgery during COVID-19 pandemic. European Annals of Otorhinolaryngology, Head Neck [Epub ahead of print, Apr 11, 2020]

11. Cheng X, Liu J, Li N, et al. Otolaryngology Providers Must Be Alert for Patients with Mild and Asymptomatic COVID-19. Otolaryngology-Head Neck Surgery. Article Commentary, April 14, 2020.

12. Bai Y, Yao L, Wei T et al. Presumed Asymptomatic Carrier Transmission of COVID-19. JAMA. 2020;323(14):1406-1407.

13. Al-Tawfiq. Asymptomatic coronavirus infection: MERS-CoV and SARS-CoV-2 (COVID-19). Travel Medicine and Infectious Disease. [Epub ahead of print, Feb 24, 2020].

14. Day M. COVID-19: four fifths of cases are asymptomatic, China figures indicate. BMJ. 2020:369:1375.

15. Lai CC, Liu YH, Wang CY, et al. Asymptomatic carrier state, acute respiratory disease, and pneumonia due to severe acute respiratory syndrome coronavirus 2 (SARS-CoV-2): Facts and myths. J Microbiol Immunol Infect. [Epub ahead of print, Mar 4, 2020]

16. Vukkadala N, Qian J, Hoslinger C, Patel ZM, Rosenthal E. COVID-19 and the Otolaryngolist: Preliminary Evidence-Based Review. Laryngoscope. [Epub ahead of print, Mar 26, 2020]

17. Zitek T. The appropriate use of testing for COVID-19. Western Journal of Emergency Medicine. 2020;21(3):471-472.

18. Ren X, Liu Y, Chen H, et al. Application and optimization of RT-PCR in diagnosis of SARS-CoV-2 infection. medRxiv. 2020.h ttps://www.medrxiv.org/content/10.1101/2020.02.25.20027755v2. Accessed May 2, 2020.

19. Wang W, Xu Y, Gao R. Detection of SARS-CoV-2 in Different Types of Clinical Specimens. JAMA. [Epub ahead of print, Mar 11, 2020]

20. Center for Disease Control. Coronavirus Disease 2019: Symptoms. https://www.cdc.gov/coronavirus/2019-ncov/symptoms-testing/symptoms.html. Accessed May $6,2020$.

21. Lei S, Jiang F, Su W, et al. Clinical characteristics and outcomes of patients undergoing surgeries during the incubation period of COVID-19 infection. EClinical Medicine. April 5, 2020, doi.org/10.1016/jeclinm.2020.200331

22. Tahamtan A \& Ardebili A. Real-time RT-PCR in COVID-19 detection: issues affecting the results. Expert Review Molecular Diagnostics. 2020;20(5):453-454.

23. Lin F, Shen Y, Fang C, et al. Incididenc of and Factors Associated with False Positives in Laboratory Diagnosis of Norovirus Infection by Amplification of the RNA-Dependent RNA Polymerase Gene. PLOS One. 2014;9(9):1-7.

24. Wollants E \& Van Ranst M. Detection of false positives with a commonly used Norovirus RT-PCR primer set. Journal Clinical Virology. 2013;56(1):84-85.

25. Alm E, Lesko B, Lindegren G, et al. Universal Single-Probe RT-PCR Assay for Diagnosis of Dengue Virus Infections. PLOS. Published December 18, 2014.

26. Kim H, Hong H, Yoon SH. Diagnostic Performance of CT and Reverse Transcriptase-Polymerase Chain Reaction for Coronavirus Disease 2019: A Meta-Analysis. Radiology. [Epub ahead of print, Apr 17, 2020]

27. Hao W \& Li M. Clinical diagnostic value of CT imaging in COVID-19 with multiple negative RT-PCR testing. Travel Medicine and Infectious Disease. [Epub ahead of print, Mar 13, 2020]

28. Ai T, Yang Z, Hou H, et al. Correclation of Chest CT and RT-PCR Testing in Coronavirus Disease 
2019 (COVID-19) in China: A report of 1014 Cases. Radiology. [Epub ahead of print, Feb 26, 2020]

29. Feng H, Liu Y, Lv M, Zhong J. A case report of COVID-19 with false negative RT-PCR test: necessity of chest CT. Jpn J Radiol. 2020;38(5):409-410.

30. Meng H, Xiong R, He R, et al. CT imaging and clinical course of asymptomatic cases with COVID-19 pneumonia at admission in Wuhan, China [published online ahead of print, 2020 Apr 12]. J Infect . 2020;S0163-4453(20)30211-5. doi:10.1016/j.jinf.2020.04.004

31. Hu Z, Song C, Xu C, et al. Clinical characteristics of 24 asymptomatic infections with COVID19 screened among close contacts in Nanjing, China. Sci China Life Sci . 2020;63(5):706-711. doi:10.1007/s11427-020-1661-4

\section{Hosted file}

Table_1_Targets.docx available at https://authorea.com/users/312328/articles/452508false-positive-rt-pcr-screening-for-sars-cov-2-in-the-setting-of-urgent-head-and-necksurgery-and-otolaryngologic-emergencies-during-the-pandemic-clinical-implications

\section{Hosted file}

Table_2_RTPCR.docx available at https://authorea.com/users/312328/articles/452508-falsepositive-rt-pcr-screening-for-sars-cov-2-in-the-setting-of-urgent-head-and-necksurgery-and-otolaryngologic-emergencies-during-the-pandemic-clinical-implications

\section{Hosted file}

Table_3_RTPCR.docx available at https://authorea.com/users/312328/articles/452508-falsepositive-rt-pcr-screening-for-sars-cov-2-in-the-setting-of-urgent-head-and-necksurgery-and-otolaryngologic-emergencies-during-the-pandemic-clinical-implications 